\title{
$R$ bits user selection switch feedback for zero forcing MU-MIMO based on low rate codebook
}

\author{
Shiyuan Li', Qimei Cui, Xiaofeng Tao and Xin Chen
}

\begin{abstract}
Channel feedback for multi-user (MU)-multiple-input multiple-output (MIMO) has been widely studied and some results have been got with random vector quantization scheme. However, while the low rate fixed codebook feedbacks are adopted, the performance of zero forcing (ZF) MU-MIMO will decrease as the unpredictable interuser interference is introduced because of quantized channel state information (CSI). To decrease inter-user interference in low rate fixed codebook feedback, an enhanced user selection switch (USS) feedback scheme for ZF MU-MIMO is proposed in this article. In USS feedback, the extra USS information is added after quantized CSI and received signal-to-noise ratio feedback. The USS information indicates inter-user interference and it can be used in user selection procedure to avoid large inter-user interference. Simulation results show that the proposed USS feedback scheme is efficient to solve the problems of unpredictable inter-user interference in conventional feedback scheme with low rate codebook in ZF MU-MIMO.
\end{abstract}

Keywords: MU-MIMO, feedback, user slection, user pairing

\section{Introduction}

It is well known that multiple-input multiple-output (MIMO) can make full use of spatial diversity and enhance data rate by spatial multiplexing. In rich scattering environment, the data rates increase linear with the minimal antenna number of the base station (BS) and user equipment (UE) compared to the single-input single-output (SISO) scheme [1]. Usually, BS equips more antennas than UE, so the spatial diversity of MIMO system is not fully utilized. To overcome this drawback, the multi-user MIMO (MU-MIMO) technique is introduced. In downlink MU-MIMO transmission, the data streams of multiple UEs are simultaneously transmitted from BS to UEs at same time and frequency resource. Each UE demodulates its data only by his own channel state information (CSI) and the data of other UEs are treated as interference.

While BS and UEs know the perfect CSI, "Dirty Paper Coding" (DPC) [2-6] is known to achieve the capacity of the MIMO downlink channel, but DPC has very high complexity to be realized in actual system. To reduce

\footnotetext{
* Correspondence: buptlishiyuan@gmail.com

Wireless Technology Innovation (WTI) Institute, Key Laboratory of Universal Wireless Communications, Ministry of Education, Beijing University of Posts and Telecommunications (BUPT), Beijing, P.R. China
}

the complexity of coding, zero forcing (ZF) [7-10] is proposed as the sub-optimal solution and the performance of ZF is close to DPC in many scenarios [11].

ZF technique needs CSI between BS and UEs while performing user selection and computing precoding matrix. The exact CSI can be got by channel reciprocity in TDD system. However, BS only can get quantized CSI by UE feedback in FDD system because the feedback channel has limited rate. So, the signals of paired UEs cannot be perfectly separated by ZF precoding and UE will receive the unwished signals of other paired UEs which is called inter-user interference. Hence, the MU-MIMO performance will be decreased with the quantized CSI in FDD system $[12,13]$. Some important conclusions with limited feedback for MU-MIMO have been got [14-19], and these studies show that the quantization bit scales linear with number of transmit antennas and logarithmic with received SNR of UE while a constant performance gap are hold compare to perfect-CSI.

In former research, the derivation of sum-rate is based on the assumption of random vector quantization (RVQ), which means the codebook of each UE is randomly generated and they are uniformly distributed on 
the unit sphere. There are some disadvantages for RVQ scheme in the actual communication system:

(1) It needs a great deal feedback bits in the case of high SNR and large number of transmit antennas [16-18]. For example, while SNR is $10 \mathrm{~dB}$ with 4 transmit antennas, it needs about 14 bits (16,384 codebooks) and while SNR is $20 \mathrm{~dB}$ with 8 transmit antennas, it needs about 35 bits $(34,359,738,368$ codebooks).

(2) The codebook needed in RVQ scheme should randomly be generated by UE before CSI feedback, and then the codebook is sharing with BS through feedback channel. So, the large codebook number will also increase feedback overhead of codebook sharing, the computational complexity of codebook generation, and cache costs of codebook storage.

(3) RVQ needs different quantized bits for different SNR cases, so it will bring some design problems. For examples, if the feedback bits are fixed, it will cause waste for low SNR case and not enough for high SNR case. If feedback bits are flexible, new codebook will be retransmitted while SNR changed and it will decrease the effects of user selection between UEs with different SNR.

Moreover, most of the current communication system adopt small codebook size and fixed codebook structure, which both known by UE and BS, to reduce the system complexity feedback overhead. In this feedback scheme, the former performance analysis for RVQ will be not suitable. In low rate fixed codebook feedback scheme, the interference between paired users is the key problem and conventional feedback and user selection scheme have on mechanism to avoid large inter-user interference. To overcome this drawback in low rate fixed codebook feedback scheme, the reasons of large interuser interference are analyzed detailed and an enhanced scheme named user selection switch (USS) feedback is proposed here. The USS feedback adds some extra information besides CSI and SNR to show the inter-user interference while performing ZF MU-MIMO transmission. With USS information, BS can avoid large interuser interference in MU-MIMO transmission in user selection procedure and enhance MU-MIMO performance.

The rest of the article is organized as follows. Section 2 introduces conventional MU-MIMO transmission model and analyzes the problem of low rate fixed codebook feedback scheme. Section 3 proposes USS feedback to enhance MU-MIMO performance and gives related user selection procedure. Section 4 gives the numerical simulation to verify the performance enhancement. Section 5 provides some conclusions.

\section{System model}

In this article, the single cell MIMO downlink channel is considered, in which the transmitter has $M$ antennas and each UE has 1 antenna. Each user only receives one data stream, and at most $M$ users can be communicated at the same time. The system model is shown in Figure 1. In conventional feedback, only SNR and CSI are fed back to BS.

The signal received by a single user $i$ can be represented as

$$
y_{i}=\sqrt{g_{i}} H_{i} x_{i}+\sum_{j \neq i} \sqrt{g_{i}} H_{i} x_{j}+n_{i}
$$

where $g_{i}$ is pathloss between $\mathrm{BS}$ and $\mathrm{UE}_{\dot{v}} H_{i} \in C^{1 \times M}$ is the normalized channel matrix between $\mathrm{BS}$ and $\mathrm{UE}_{i}$, $x_{i}$ is the transmitted signals with an average power constraint $E\left\{\left\|x_{i}\right\|^{2}\right\}=P_{i},\|\cdot\|$ stands for norm operator, $P_{i}$ is the power constraint of each user's data stream, $n_{i}$ is the additive white Gaussian noise with $\sigma^{2}$ variance, and $y_{i}$ is the signal received by $\mathrm{UE}_{i}$.

The procedure of conventional ZF MU-MIMO is as follows $[10,18]$.

\subsection{Quantized CSI feedback}

It assumed that each user knows perfect CSI and normalized it to a unit norm vector $H_{i}$. The quantization vector is chosen from a fixed codebook of size $N=2^{B}$

$$
C=\left\{c_{1} \cdots c_{N}\right\}, \quad\left(c_{j} \in C^{1 \times M}, N=2^{B}\right) .
$$

The codebook $C$ is designed offline and both known to the BS and UE. UE will select a vector from codebook according to the minimum distance criterion as following equation,

$$
k=\arg \max _{1 \leq j \leq N}\left\|H_{i} c_{j}^{H}\right\| .
$$

Then the index $k$ is fed back to BS, and BS treats $w_{i}=$ $c_{k}$ as the channel matrix $H_{i}$ of $\mathrm{UE}_{i}$.

\subsection{SNR Feedback}

Each user will feed back its received SNR with assumption of single user transmission. The SNR of users is

$$
S N R_{i}=\frac{\left\|\sqrt{g_{i}} H_{i} x_{i}\right\|^{2}}{\sigma^{2}}=g_{i} P_{i} / \sigma^{2} .
$$

UE can measure it by reference signals (RS), as the RS sequence and its power are known to UE. In the practical system, this information is quantized with small number of bits. In order to concentrate on the effect of CSI quantization and user selection, it assumes that the SNR is directly fed back without quantization.

\subsection{User selection}

After BS received feedback, it will select some paired users from serving user set $U=\left\{\mathrm{UE}_{1}, \ldots, \mathrm{UE}_{K}\right\}$, which is 


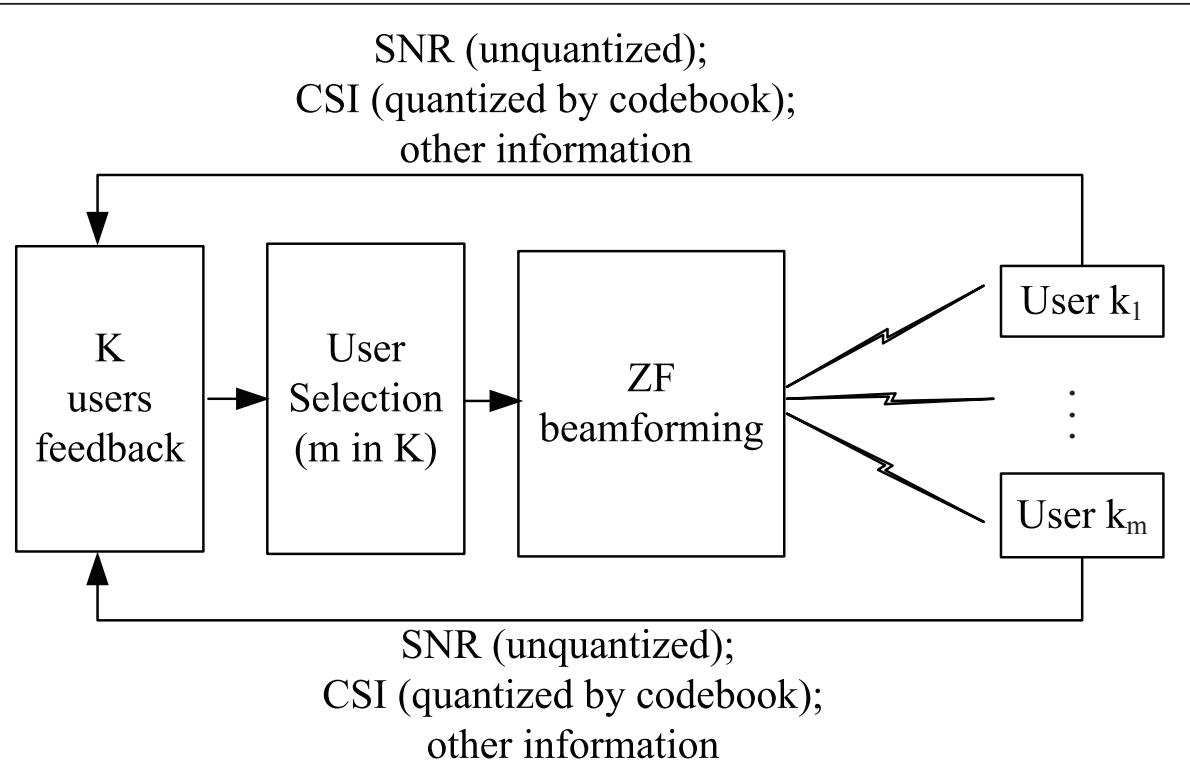

Figure 1 Downlink MU-MIMO system

correspond to all the users served by BS. The number of selected users is determined by higher layer and must be no more than $m$ which is the number of transmit antennas. There have been many proposed user selection criteria [20-25] and the basic principle is to maximize the total throughputs of the paired users. It is known that in MIMO transmission, the higher throughput will be gotten with the smaller channel correlation between paired users. So, in the simulation of conventional MUMIMO in the article, BS will select users which have the minimal spatial channel correlation between each other. That's means the maximum correlation between selected users will be minimal in all possible MUMIMO user combinations. The user selection criterion can be expressed as

$$
\min _{V} \max _{i, j \in V ; i \neq j}\left|H_{i} H_{j}^{H}\right|,
$$

where $|\cdot|$ stands for absolute value, $(\cdot)^{H}$ stands for Hermite transpose, $V$ is paired user set in which the users are scheduled together to form MU-MIMO.

\subsection{ZF precoding}

After the paired user set $V$ is determined, BS will calculate the precoding matrix for these paired users. The precoding matrix is computed by ZF methods:

$$
\left(p_{1} \cdots p_{M}\right)=\left(\begin{array}{c}
w_{1} \\
\vdots \\
w_{M}
\end{array}\right)^{+}
$$

where $p_{i}$ is precoding vector of $\mathrm{UE}_{i}, w_{i}$ is the quantized CSI of $\mathrm{UE}_{i},(\cdot)^{+}$stands for pseudo-inverse operation.

So, the received signals of uses in set $V$ are

$$
\left(\begin{array}{c}
y_{1} \\
\vdots \\
y_{M}
\end{array}\right)=\left(\begin{array}{c}
\sqrt{g_{1}} H_{1} \\
\vdots \\
\sqrt{g_{M}} H_{M}
\end{array}\right)\left(p_{1} \cdots p_{M}\right)\left(\begin{array}{c}
x_{1} \\
\vdots \\
x_{M}
\end{array}\right)+\left(\begin{array}{c}
n_{1} \\
\vdots \\
n_{M}
\end{array}\right) .
$$

Here, the total power should be reallocated among multiple users' data stream. The power adjustment includes coefficient scaling of users' precoding vector and power allocation of users' data stream. The received signals of users change to following equation:

$$
\left(\begin{array}{c}
y_{1} \\
\vdots \\
y_{M}
\end{array}\right)=\left(\begin{array}{c}
\sqrt{g_{1}} H_{1} \\
\vdots \\
\sqrt{g_{M}} H_{M}
\end{array}\right)\left(\frac{p_{1}}{\alpha_{1}} \cdots \frac{p_{M}}{\alpha_{M}}\right)\left(\begin{array}{c}
\beta_{1} s_{1} \\
\vdots \\
\beta_{M} s_{M}
\end{array}\right)+\left(\begin{array}{c}
n_{1} \\
\vdots \\
n_{M}
\end{array}\right)
$$

where $\alpha_{i}$ is coefficient scaling factor, $\beta_{i}$ is power allocation factor, and $s_{i}$ is transmit symbols with unit variance. The total power should be no more than max transmit power $P_{\text {total }}$, and the constraint is

$$
\sum_{i=1}^{M} \frac{\beta_{i}^{2}}{\alpha_{i}^{2}}\left\|p_{i}\right\|^{2}=P_{\text {total }}
$$

The received signal of each user is

$$
y_{i}=\sqrt{g_{i}} H_{i} \frac{\beta_{i}}{\alpha_{i}} p_{i} s_{i}+\sqrt{g_{i}} H_{i} \sum_{j=1, j \neq i}^{M} \frac{\beta_{j}}{\alpha_{j}} p_{j} s_{j}+n_{i},
$$


where $\sqrt{g_{i}} H_{i} \frac{\beta_{i}}{\alpha_{i}} p_{i} s_{i}$ is wanted signal and $\sqrt{g_{i}} H_{i} \sum_{j=1, j \neq i}^{M} \frac{\beta_{j}}{\alpha_{j}} p_{j} s_{j}$ is inter-user interference.

2.5. MU-MIMO performance with conventional feedback The user SNR of MU-MIMO is

$$
\text { MU_SNR }_{i}=\frac{g_{i} \frac{\beta_{i}^{2}}{\alpha_{i}^{2}}\left\|H_{i} p_{i}\right\|^{2}}{\sum_{j=1, j \neq i}^{M} g_{i} \frac{\beta_{j}^{2}}{\alpha_{j}^{2}}\left\|H_{i} p_{j}\right\|^{2}+\sigma_{i}^{2}} .
$$

The total throughput is

$$
R_{\mathrm{MU}}=\sum_{i=1}^{m} \log \left(1+\mathrm{MU} \mathrm{SNR}_{i}\right) .
$$

\subsection{The problems of conventional feedback}

In the conventional feedback scheme, BS and UE cannot know the MU_SNR clearly. For $\mathrm{UE}_{i}$, it knows its channel matrix $H_{i}$, but does not know the channel of paired users. For BS, it knows paired users, but does not know exact channel matrix of UEs. So, the $\left\|H_{i} p_{j}\right\|^{2}$ cannot be known for BS and UE. Hence, the transmitting rate $R$ is evaluated in conventional user selection.

Usually $R$ is evaluated with the assumption of no inter-user interference, which means $\left\|H_{i} p_{j}\right\|^{2} \approx 0$. But for the paired user, the inter-user interference may be very large and lead the performance decrease heavily, while $\left\|H_{i} p_{j}\right\|^{2} \gg 0$. In user pairing, BS does not know the exact inter-user interference, so it has no mechanism to avoid large inter-user interference in user selection criteria.

The large inter-user interference will decrease throughput largely. For example, if the inter-user interference $\left\|H_{i} p_{j}\right\|^{2}$ is more than 0.0833 in the configuration of $2 \mathrm{Tx}, 2$ paired UE, $10 \mathrm{~dB}$ SNR, the sum rate of MU-MIMO will less than SISO transmission. And the inter-user interference should be smaller in high SNR region than in low SNR region. Unfortunately, the interuser interference usually is not small enough for MUMIMO requirement in low fixed codebook scheme. Figure 2 shows the CDF of inter-user interference with 4 bits DFT codebook while the quantized CSI of paired user is orthogonal. It can be seen that about $50 \%$ of inter-user interference are more than 0.1 ; so many users are paired with large inter-user interference. Although the MU-MIMO will not work well with the large interuser interference, the conventional feedback and user selection method cannot provide enough information to distinguish large inter-user interference and small interuser interference.
These will cause two serious problems:

(1) The performance gain of MU-MIMO will decrease, especially in high SNR case. Figure 3 shows the MUMIMO (two paired users) performance of 4 bits feedback with DFT codebook, compared to SISO case and perfect CSI feedback. It can be seen that MU-MIMO with perfect CSI feedback has very high rate about double of that in SISO case. But for low rate quantized feedback (4 bits), the performance gain falls largely compare to perfect CSI feedback, as the CSI is the quantized version with low codebook size. The performance gain is little at high SNR region because the inter-user interference of paired users is randomly in quantized feedback with conventional user selection methods, and MU-MIMO performance is sensitive to inter-user interference in high SNR case.

(2) While the quantized bits increase, the performance enhancement may not be obvious for some codebook types. Figure 4 shows the sum data rate of MU-MIMO quantized with DFT codebook of different bits. It can be seen that while the number of quantized bits increase from 2 to 3 bits the performance enhancement is obvious, and performance enhancement is little while number of quantized bits increase from 3 to 6 bits. Concluded from the growth trend, when the number quantized bit is more than 6 bits, the performance is near to case of 6 bits. So, increasing codebook size is no use to enhance MU-MIMO performance. The reason is that the increasing number of quantized bits cannot decrease the inter-user interference of paired users for fixed codebook structure unlike RVQ feedback scheme.

\section{Algorithm}

To decrease the bad effect of random inter-user interference in low rate fixed codebook feedback scheme, a novel USS feedback scheme is proposed. In the USS feedback, extra USS information is added after CSI feedback to show the inter-user interference. And this information is used in user selection algorithm to avoid large inter-user interference. The detailed process of the proposed scheme is elaborated as follows.

\subsection{Grouping quantized codebook}

In MU-MIMO transmission, the paired users are usually selected with small correlation between their channels. In USS feedback scheme, codebook $C$ is divided into several groups, and only the users whose quantized CSI from the same group can be paired together. The codebook $C$ is divided as follows:

$$
C_{k}=\left\{c_{k 1}, \ldots, c_{k l}\right\}\left(\left\|c_{k i} H_{k j}^{H}<R\right\| ; k i \neq k j ; k i, k j, k=1, \ldots, N\right),
$$

where $C_{k}$ is subset of codebook $C$ satisfied $C=\underset{k=1, \ldots, m}{\cup} C_{k}$ and $C_{k 1} \cap C_{k 2}=\varnothing(k 1 \neq k 2), c_{k i}$ is element of 


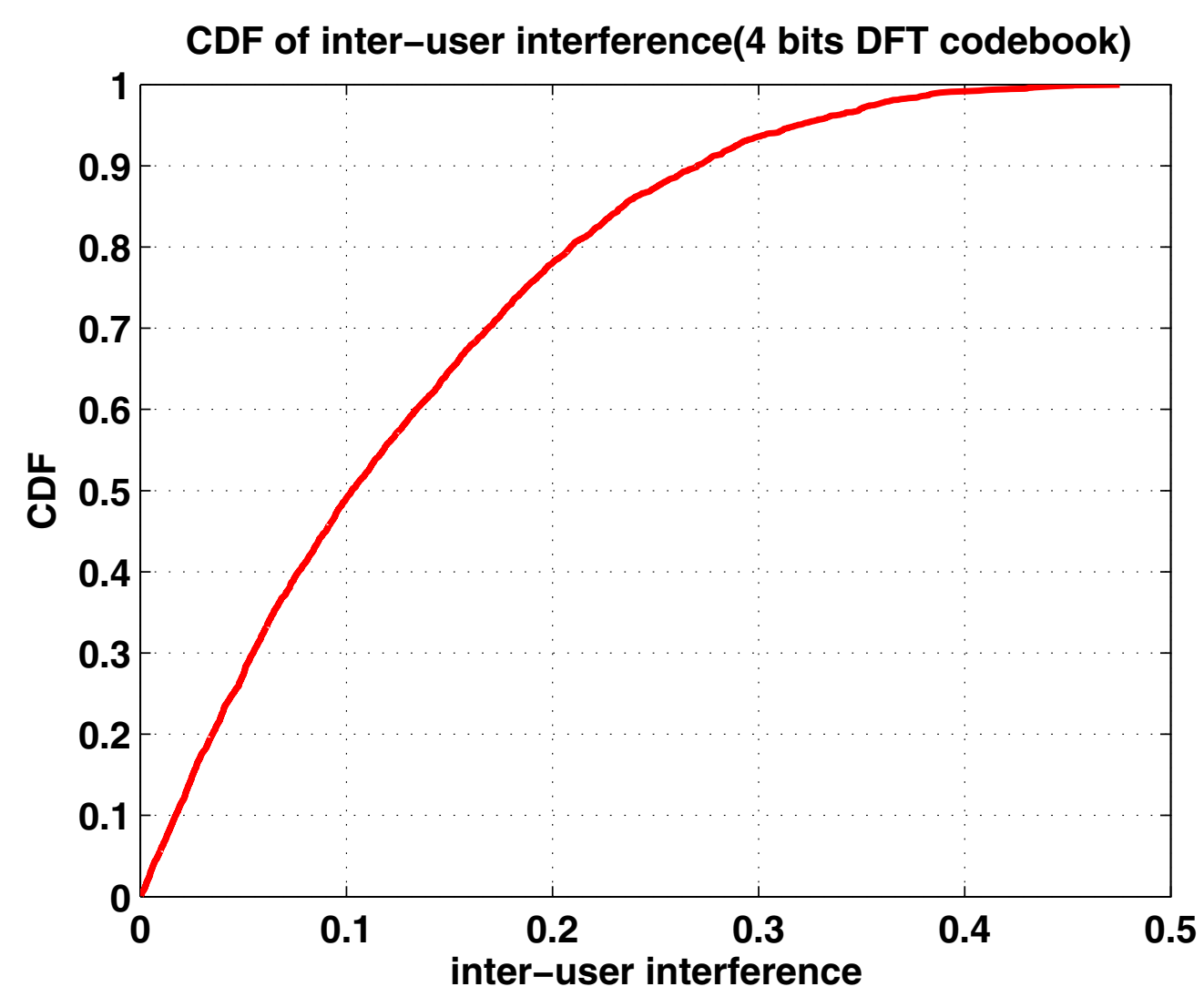

Figure 2 CDF of inter-user interference (4 bits DFT codebook)

codebook $C, m$ is number of groups, $l$ is element number of subset, $N$ is codebook size with the relevance $N=$ $l " m, R$ is correlation threshold between code vector in subset, which means the correlation between any two paired users are no more than $R$.

Only the users which their feedback belong to same group can be paired together, so the correlation between any two paired users are no more than $R$. At most $M$ users can be transmit at same time in MU-MIMO, so lets $l \geq M$, and all the $M$ users can be selected in the same set. In the simulation of this article, the DFT codebook is adopted with setting $l=M$ and $r=0$, as DFT codebooks are naturally separated into orthogonal groups, which has $M$ orthogonal vectors.

\subsection{USS information feedback}

In USS feedback scheme, $(l-1)^{*} r$ additional bits named USS information are fed back to BS besides CSI and SNR, and this information is used to indicate the MU-MIMO performance. In sub-codebook groups, user can be paired with other ( $l-1)$ vector, so USS information uses $r$ bit(s) for each vector to show the MU-MIMO performance while user is paired with this vector. The feedback contents are $\left(\mathrm{USS}_{1}, \ldots, \mathrm{USS}_{i-1}\right)$ and $\mathrm{USS}_{i}$ corresponding to the $i$ th vector in sub-codebook except the vector which user is fed back. For example, if $r=1$, the user can be paired with $i$ th vector while $\mathrm{USS}_{i}=1$, and the user cannot be paired with $i$ th vector while $\mathrm{USS}_{i}=0$.

The value of USS information is relative to transmission and feedback configuration, such as number of paired user $m$ and USS information bits $r$. The details of the value calculation will be shown in Section 3.4 for different configurations.

\subsection{User selection procedure}

In USS feedback scheme, the user selection will use USS information to avoid large inter-user interference. The step is as follows:

(1) BS defines three sets: serving user set $U=\left\{\mathrm{UE}_{1}, \ldots\right.$, $\left.\mathrm{UE}_{K}\right\}$, corresponding to all the users served by BS; (2) user CSI set $W=\left\{w_{1}, \ldots, w_{K}\right\}$, corresponding to users' CSI; (3) paired user set $\mathrm{MU}=\varnothing$, corresponding to the users scheduled together to adopt MU-MIMO. BS sets the number of paired users (more than 1 and no more than the number of transmit antennas).

(2) BS selects first two users $(i, j)$ from set $U$. The $\mathrm{UE}_{i}$ and $\mathrm{UE}_{j}$ should satisfy the conditions: (a) their CSI feedback should be in the same codebook group $C_{k}$, that 


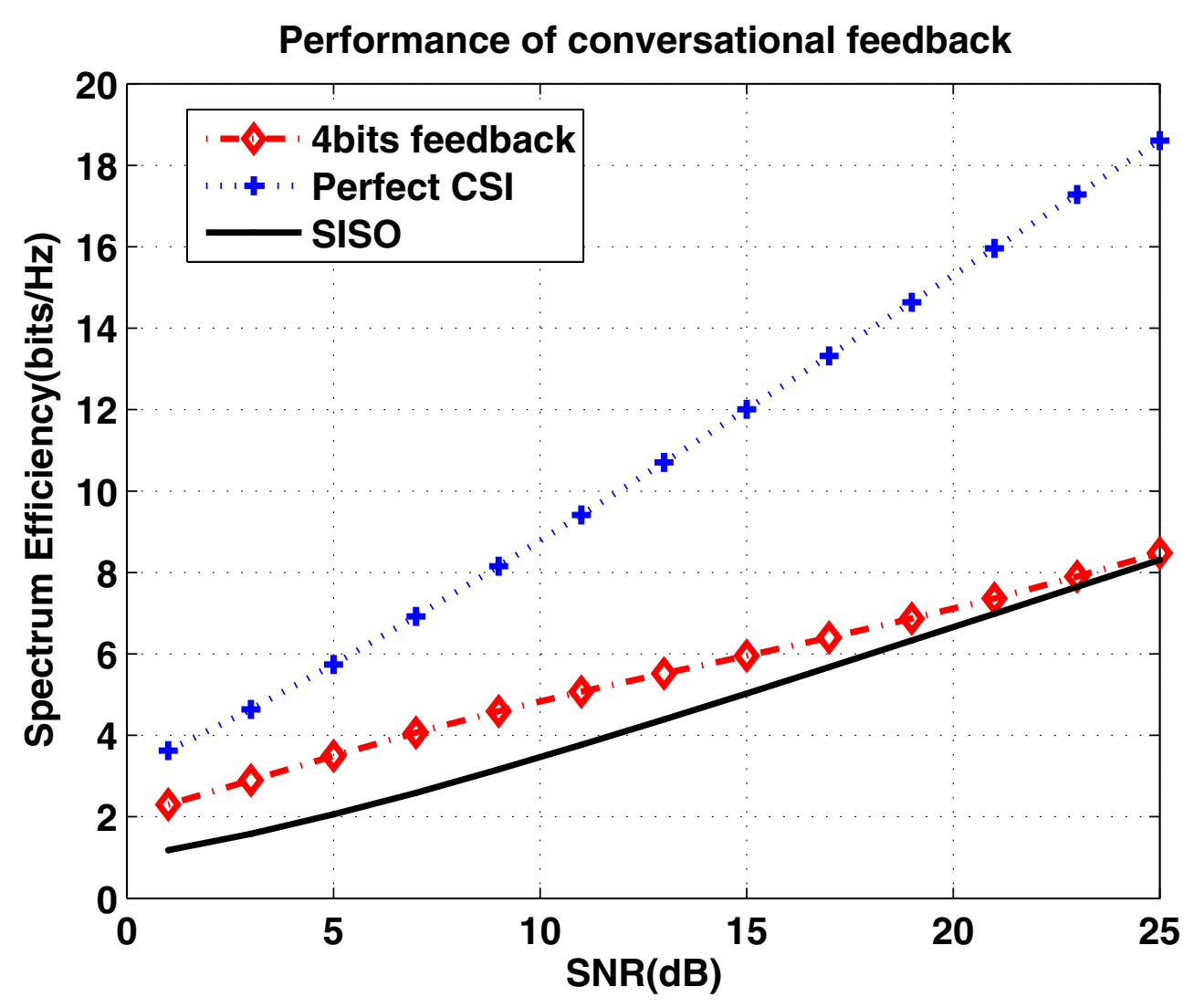

Figure 3 MU-MIMO performance comparison.

means $w_{i}, w_{j} \in C_{k}$; (b) the USS information for paired vector should not be equal to zero, that means $\left(\mathrm{USS}_{i l 1}>\right.$ $0, \mathrm{USS}_{j l 2}>0, c_{k l 1}=w_{j}, c_{k l 2}=w_{j}$ ); (c) the summation of USS information for paired vector should be maximum in all users which satisfy conditions (a) and (b), that means $(i, j)=\max _{\mathrm{UE}_{i}, \mathrm{UE}_{j} \text { satisfy (a) and (b) }}\left(\mathrm{USS}_{i l 1}+\mathrm{USS}_{j l 2}\right)$.

If the two users can be found, BS will put them into paired user set $\mathrm{MU}=\left\{\mathrm{UE}_{i}, \mathrm{UE}_{j}\right\}$, and remove them from serving user set $U=U-\left\{\mathrm{UE}_{i}, \mathrm{UE}_{j}\right\}$. Otherwise, user pairing will be stopped and single user mode will be adopted.

(3) If the number of paired user is enough, start ZF procedure to compute precoding matrix. Otherwise, select the next user $o$ from set $U$. The $\mathrm{UE}_{o}$ should satisfy the conditions: (a) its CSI feedback should be in codebook group $C_{k}$, same to users in set $\mathrm{MU}$, that means $w_{o} \in C_{k}$; (b) the USS information for paired vector of $\mathrm{UE}_{o}$ and users in set MU should be more than zero, that means USS $_{\text {oli }}>0$, $\left.\mathrm{USS}_{i l o}>0, c_{k l i}=w_{i} c_{i l o}=w_{o}, \mathrm{UE}_{i} \in \mathrm{MU}\right)$; (c) the summation of USS information for paired vector should be maximum in all users which satisfy conditions (a) and (b), that

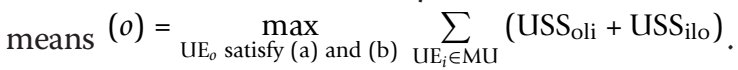

If the user $o$ can be found, BS will put it into paired user set $M U=M U+\left\{U_{o}\right\}$, and remove them from serving user set $U=U-\left\{\mathrm{UE}_{o}\right\}$. Otherwise, user pairing will be stopped and start ZF procedure to compute precoding matrix for the users in set MU.

(4) If the number of paired user is enough, start ZF procedure to transmit users' data. Otherwise, go to step 3 to select another user.

\subsection{USS value calculation}

The value of USS information is relative to the number of paired user $m$ and USS information bits $r$. In this section, different cases will be discussed separately.

(a) $r=1$ and $m=2$

For two paired users, the SNR for each user can get from Equation (11),

$$
\operatorname{MU\_ SNR}_{i}=\frac{g_{i} \frac{\beta_{i}^{2}}{\alpha_{i}^{2}}\left\|H_{i} p_{i}\right\|^{2}}{g_{i} \frac{\beta_{j}^{2}}{\alpha_{j}^{2}}\left\|H_{i} p_{j}\right\|^{2}+\sigma_{i}^{2}},
$$




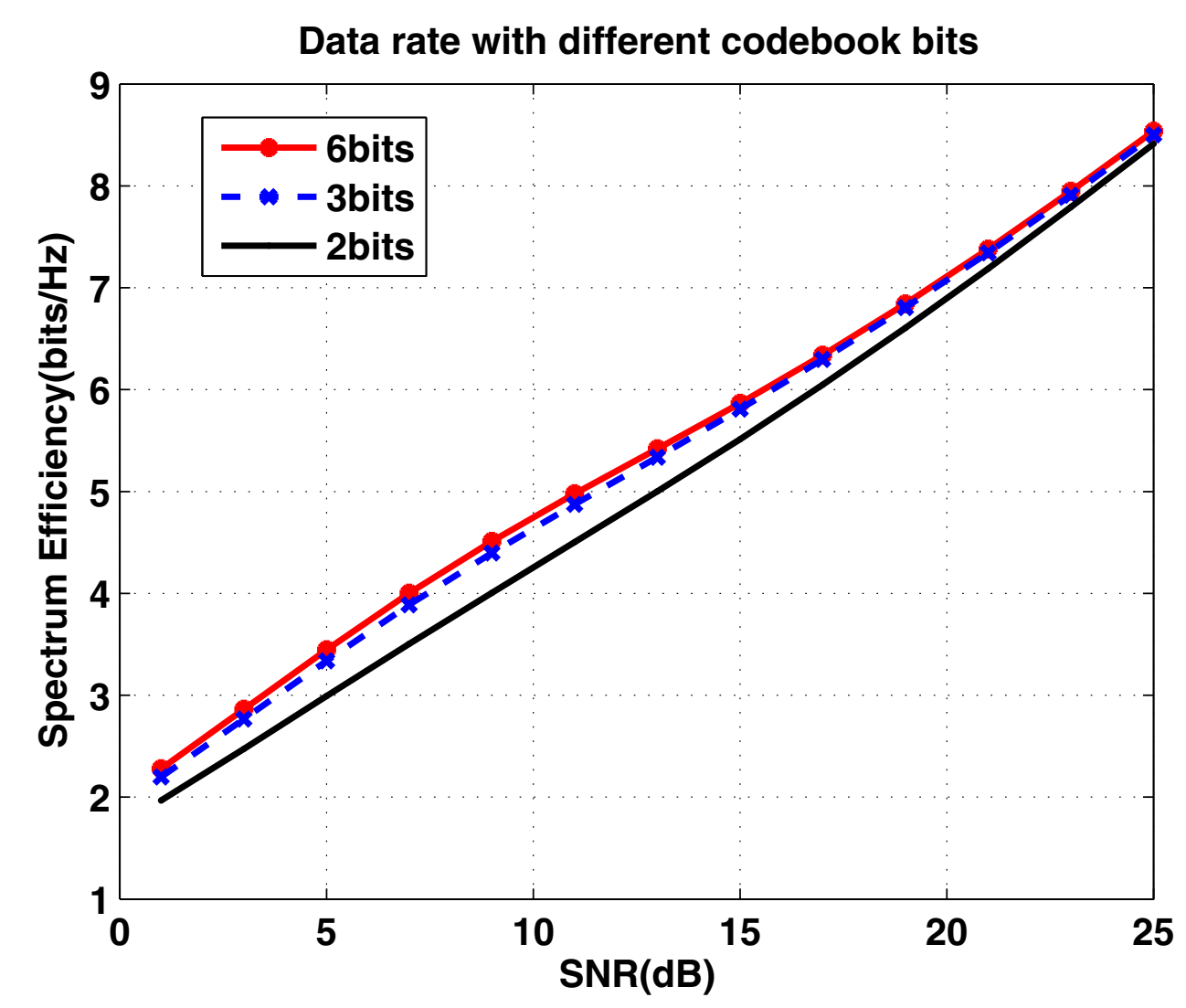

Figure 4 Data rate with different codebook bits

where $\alpha_{i}$ is coefficient scaling factor, $\beta_{i}$ is power allocation factor. The total power should no more than max transmit power $P_{\text {total }}$, and the constraint is $\frac{\beta_{1}}{\alpha_{1}}\left\|p_{1}\right\|^{2}+\frac{\beta_{2}}{\alpha_{2}}\left\|p_{2}\right\|^{2}=P_{\text {total }}$.

The precoding vector can be gotten from Equation (6),

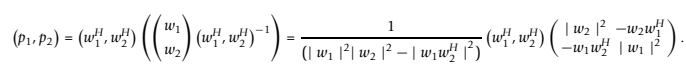

Define the correlation of vector: $w_{1} w_{2}^{H}=\sigma e^{j \phi}$. So, the precoding matrix changes to

$$
p_{i}=\frac{\left|w_{j}\right|^{2} w_{i}^{H}-\sigma e^{-j \phi} w_{j}^{H}}{\left(\left|w_{i}\right|^{2}\left|w_{j}\right|^{2}-\sigma^{2}\right)} \quad(i \neq j)
$$

Each user knows its channel matrix and the vector of paired user is selected in subset $C_{k}$. So, user can calculate the exact SNR of MU-MIMO for each vector in set $C_{k}$.

The equation can be simplified with following assumptions: (1) usually the codebook is normalize vector, that means $\left\|w_{i}\right\|^{2}=1$; (2) normalize precoding vector for each users, that means $\left\|p_{i}\right\|^{2}=\alpha_{i}^{2}$; (3) power is equally allocated in the paired users, that means $\beta_{i}^{2}=P_{\text {total }} / m$, where $m$ is number of paired users; (4) define correlation of CSI quantized as $H_{i} w_{i}^{H}=a_{i} e^{j \phi_{a i}}$; (5) define inter-user interference as $H_{i} w_{j}^{H}=b_{i j} e^{j \phi_{b i j}}(i \neq j)$. By substituting Equation (16) into Equation (14), we can get

$$
\text { MU_SNR }_{i}=\frac{\frac{g_{i} P_{\text {total }}}{2\left\|p_{i}\right\|^{2}} \frac{\left|a_{i} e^{j \phi_{a i}}-\sigma e^{-j \phi} b_{i j} e^{j \phi_{b i j}}\right|^{2}}{\left(1-\sigma^{2}\right)^{2}}}{\frac{g_{i} P_{\text {total }}}{2\left\|p_{j}\right\|^{2}} \frac{\left|b_{i j} e^{j \phi_{b j i}}-\sigma e^{j \phi} a_{i} e^{j \phi_{a i}}\right|^{2}}{\left(1-\sigma^{2}\right)^{2}}+\sigma_{i}^{2}} .
$$

This result can be used in USS information calculation. In USS feedback scheme, a correlation threshold $R$ is used in codebook subset. It means in above equations that the correlation $\sigma$ must be no more than $R$ as the paired vector is selected from same subset. With different value of $R$, it can be divided into two categories:

(a-1) $R \approx 0$. In this case, it can be thought that the paired vector is orthogonal, so the correlation $\sigma$ can be 
tread as zero. The precoding matrix changed to $p_{i}=w_{i}^{H}$, and Equation (17) can be simplified as:

$$
\mathrm{MU}_{-} \mathrm{SNR}_{i}=\frac{g_{i} P_{\text {total }} a_{i}^{2} / 2}{g_{i} P_{\text {total }} b_{i j}^{2} / 2+\sigma_{i}^{2}}=\frac{a_{i}^{2}}{b_{i j}^{2}+2 / S N R_{i}},
$$

where $\mathrm{SNR}_{i}$ is the measured SNR defined in Equation (4).

So, throughput of $\mathrm{UE}_{i}$ is

$$
R_{i}=\log \left(1+\text { MU_SNR }_{i}\right)=\log \left(1+\frac{a_{i}^{2}}{b_{i j}^{2}+2 / \mathrm{SNR}_{i}}\right)
$$

Because user does not know the vector which BS will be schedule in user pairing, the actual transmit rate cannot be known. In USS feedback scheme, all the paired vectors are in one subcodebook $C_{k}=\left\{c_{k 1}, \ldots, c_{k l}\right\}$, and for one UE, the number of candidate pairing vector is $l-1$. So, for each candidate pairing vector in subcodebook, user will evaluate its throughput when this vector is selected as paired vector, and the USS information is calculated based on this evaluated throughput.

User assumes that the paired user has the same correlation of quantized CSI $a$ and the same inter-user interference level $b$, so the evaluated sum throughput is $R_{k j}=$ $2 R_{i}(j \neq i)$. If the sum throughput for the vector $c_{k j}$ is more than MISO throughput $R_{\mathrm{su}}=\log (1+\mathrm{SNR})$, set $\mathrm{USS}_{k j}=1$, which means the performance is better while $\mathrm{UE}_{i}$ paired with vector $c_{k j}$, otherwise set $\mathrm{USS}_{k j}=0$, which means the inter-user interference is large while $\mathrm{UE}_{i}$ paired with vector $c_{k j}$ and $\mathrm{UE}_{i}$ should avoid to pair with this vector.

(a-2) $R>0$. In this case, the correlation $\sigma$ should be considered. Equation (17) changed to

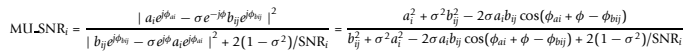

From CSI quantization criterion, it is known that $a$ is near to 1 . Usually, the correlation $\sigma$ is set near to 0 to enhance the MU-MIMO performance and the inter-user interference $b$ will be small guaranteed by user selection procedure. So, it can be thought that $\sigma b \ll a$. Equation (20) can be simplified as

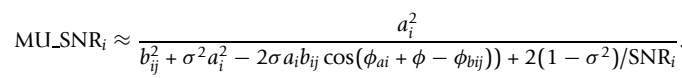

The USS information calculation is same to the case of $R \approx 0$. The difference is that $\mathrm{MU}_{-} \mathrm{SNR}_{i}$ will use Equation (21) instead of Equation (18) in USS calculation.

(b) $r=1$ and $m>2$

If more than two users are paired together to form MU-MIMO, then the SNR of MU-MIMO user will be decreased compare to two paired users, as the inter-user interference is $m-1$ times and the power allocation of each user is also decreased. It assumes that the power is equally allocated to each user and the paired users have the same correlation of quantized CSI $a$ and inter-user interference level $b$ for each paired vector.

For the case of $R \approx 0$, the evaluated MU-MIMO SNR changed to

$$
\mathrm{MU}_{-} \mathrm{SNR}_{i}=\frac{a_{i}^{2}}{(m-1) b_{i j}^{2}+m / \mathrm{SNR}_{i}} .
$$

While $R>0$, the evaluated MU-MIMO SNR changed to

$$
\mathrm{MU}_{-} \mathrm{SNR}_{i} \approx \frac{a_{i}^{2}}{(m-1)\left(b_{i j}^{2}+\sigma^{2} a_{i}^{2}-2 \sigma a_{i} b_{i j} \cos \left(\phi_{a i}+\phi-\phi_{b i j}\right)\right)+m\left(1-\sigma^{2}\right) / \mathrm{SNR}_{i}} .
$$

The evaluated sum rate changed to

$$
R_{k j}=m R_{i}=m \log \left(1+\mathrm{MU}_{-} \mathrm{SNR}_{i}\right) .
$$

The USS information calculation is same to the case of $m=2$. The difference is that MU_SNR ${ }_{i}$ uses Equations (22) and (23) for different cases and the sum throughput $R_{k j}$ uses Equation (24).

(c) $r>1$

If each USS is more than 1 bit, it should be quantized by $2^{r}$ rank. The sum throughput $R_{k j}$ is evaluated and it is mapped into region from $R_{\text {lower }}$ to $R_{\text {upper }}$ with $r$ bits. The sum rate $R_{k j}$ is calculated same to cases (a) and (b). The lower bound is defined as single user performance $R_{\text {lower }}=\log (1+\mathrm{SNR})$, as sum rate of MU-MIMO should be more than single user transmission. The upper bound is defined as the users paired with orthogonal vectors with no inter-user interference:

$$
R_{\text {upper }}=m \log \left(1+\frac{a_{i}^{2}}{(m-1)\left(\sigma^{2} a_{i}^{2}\right)+m\left(1-\sigma^{2}\right) / \mathrm{SNR}_{i}}\right) .
$$

The quantization is performed as follows:

(1) if $R_{k j} \leq R_{\text {lower, }}$, set $\mathrm{USS}_{k j}=0$;

(2) if $R_{k j} \geq R_{\text {upper }}$, set $\mathrm{USS}_{k j}=2^{r}$;

(3) if $R_{\text {lower }}<R_{k j}<R_{\text {upper }}$, set uss $k_{k j}=1+\left[\left(\frac{R_{k j}-R_{\text {lower }}}{R_{\text {upper }}-R_{\text {lower }}} * r^{r}\right)\right\rfloor$, where $L \cdot\rfloor$ is floor function.

\subsection{Feedback overhead}

In USS feedback scheme, the extra USS information is added after quantized CSI, and the feedback overhead is changed. So, the overhead of USS feedback, conventional feedback, and RVQ feedback is analyzed in this section. As discussed above, it assumed that (1) the codebook size is $N=2^{B}$; (2) the quantization vector $c_{j} \in$ $C^{1 \times M}$; (3) UE will feed back one quantized CSI in each feedback period.

For conventional feedback, only a quantized CSI is fed back to BS in each feedback period, so the feedback overhead is $B$ bits in a feedback period.

For USS feedback, in each feedback period, the extra USS information is fed back to BS besides the quantized 
CSI. As discussed in Section 3.4, it has $l$ elements in subset and $r$ bits USS information for each element in subset. So, the feedback overhead is $B+(l-1)^{*} r$ bits in a feedback period.

For RVQ feedback, a quantized CSI is fed back to BS in each feedback period. Besides, the random codebook should be shared between BS and UE, and this codebook is randomly generated by UE then fed back to BS through feedback channel. It is assumed the random codebook can be used in $q$ periods and the 16 bits quantization with short floating point number is adopted for each complex element of codebook. So, the initialization overhead is $N^{*} M^{*} 16^{*} 2$, and this overhead cover to each period is $N^{*} M^{*} 16^{*} 2 / q$. The totally feedback bits in a feedback period is $N^{*} M^{*} 32 / q+B$.

The overhead comparison of the three methods is list in Table 1.

\section{Simulation}

In this section, a MIMO system with $M=4$ transmit antennas at the BS and single antenna at the UE is considered. The DFT codebook with different size is used in simulation. DFT codebook has orthogonal vector groups, so each orthogonal vector group is treated as one subcodebook. Hence, the correlation threshold $R$ is equal to zero.

Figure 5 shows the throughput among SISO, perfect SCI feedback, conventional feedback with 7 bits CSI quantization, 4 bits RVQ feedback, and 7 bits USS feedback ( 4 bits CSI quantization +3 bits USS information). RVQ feedback needs to share random codebook between UE and BS through uplink feedback channel and this one-off overhead should be converted into each feedback period, assumed it is equal to 3 bits per feedback period (the lifetime of random codebook is about 650 feedback periods). So, the totally feedback overhead of the three feedback scheme is same. In simulation, 2 paired users are selected from total 20 users. It can be seen that the performance of RVQ feedback is higher than conventional feedback and USS feedback in low SNR region. In high SNR region, the performance gain of RVQ feedback and conventional feedback compared to SISO is decreased. Unlike the conventional feedback, the performance gain of USS feedback is nearly constant with SNR increase, so the performance gain is not decreased in high SNR region and the performance gain is about 2 bits $/ \mathrm{Hz}$. From this result, it can be seen that the proposed USS feedback scheme has better performance enhancement in high SNR region.

Figure 6 shows the throughput of two paired users which are selected from different number of users in conventional feedback with 7 bits CSI quantization, 4 bits RVQ feedback, and 7 bits USS feedback (4 bits CSI quantization +3 bits USS information). It can be seen that the performance of RVQ feedback and conventional feedback changes very small with different number of users. This is because the user selection for conventional feedback and RVQ feedback cannot avoid large interuser interference brought by channel quantization. For the USS feedback, the performance is increased with the number of users increasing. The increasing is obvious for small number of users and little for large number of users. It is because the user pairing procedure usually cannot find proper paired users for MU-MIMO transmission in small user number case. While the number of users increases, it has more users with little interuser interference, and then the user pairing procedure for USS feedback is easily to find proper paired users for MU-MIMO.

Figure 7 shows the throughput with different CSI quantization bits in USS feedback, RVQ feedback, and conventional feedback with 7 bits CSI quantization. As shown in Figure 4, the performance is almost same while the feedback bits is more than 3 , so the performance of conventional feedback with 7 bits is showed here to stand for performance of conventional feedback with different feedback bits. It can be seen that the performance is increased with the feedback bits increase in RVQ feedback, as the quantization accuracy is increased. For the USS feedback, the performance is increased with feedback bits increased because of the enhanced quantization accuracy. While the CSI quantization bits increase to 6 bits, there is a performance decreasing in high SNR region, that is, because with the CSI quantization bits increasing, the number of codebook subset is increase, so little users will be in one subset than lower CSI quantization bits. Hence, the user pairing procedure cannot find proper users to form MU-MIMO in this region, and performance will be decreased when SU transmission is adopted. From this result, it can be seen that the proposed USS feedback scheme is suitable for very low CSI quantization bits.

Figure 8 shows the throughput with case of $r>1$ in USS feedback scheme. It can be seen that the

Table 1 The overhead comparison

\begin{tabular}{lllll}
\hline Scheme & Initialization & Quantized CSI & Additional & Totally feedback bits/period \\
\hline Conventional & 0 & $B$ & 0 & $B$ \\
USS & 0 & $B$ & $(I-1)^{*} r$ & $B+(l-1)^{*} r$ \\
RVQ & $N^{*} M^{*} 16^{*} 2 / q$ & $B$ & 0 & $N^{*} M^{*} 32 / q+B$ \\
\hline
\end{tabular}




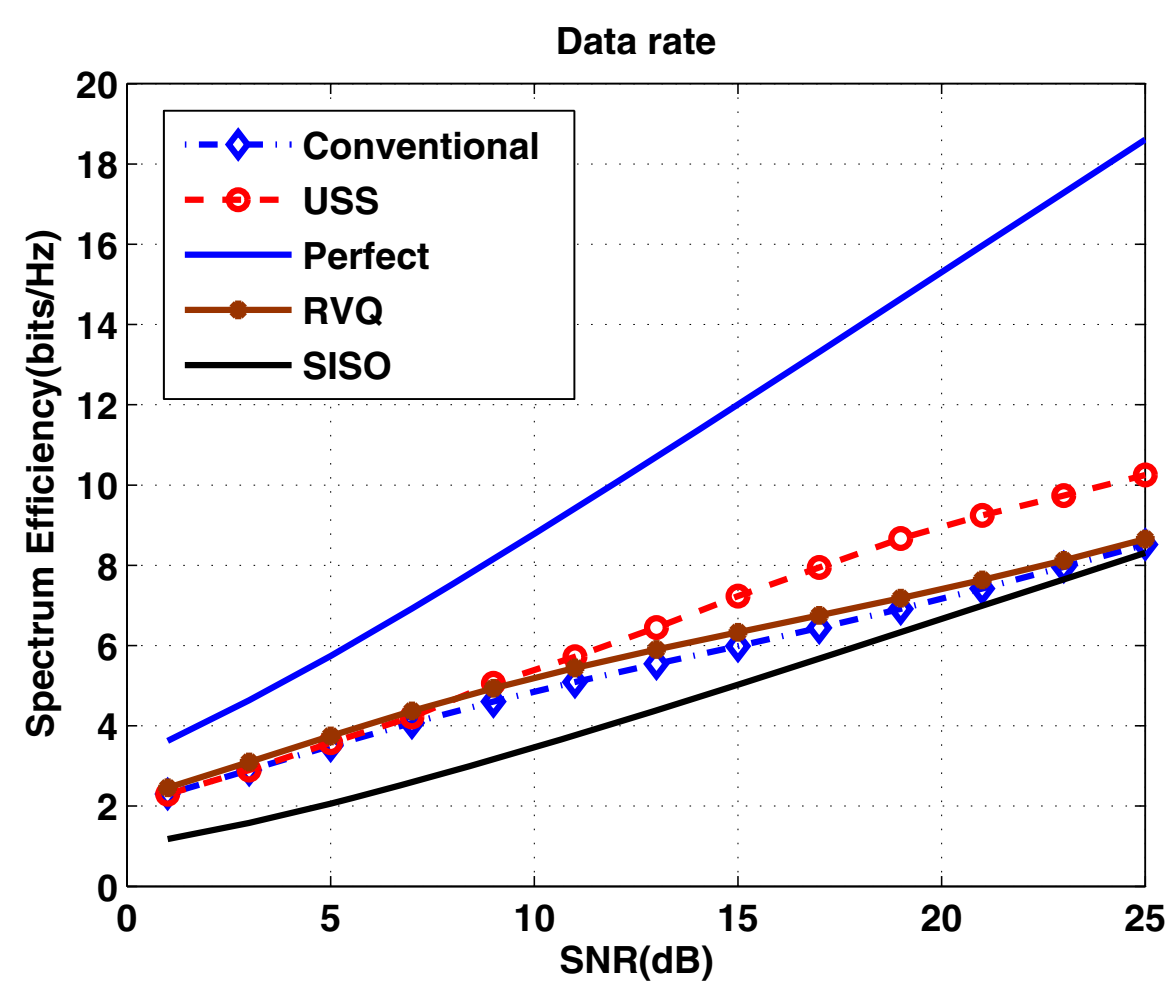

Figure 5 Date rate comparison.

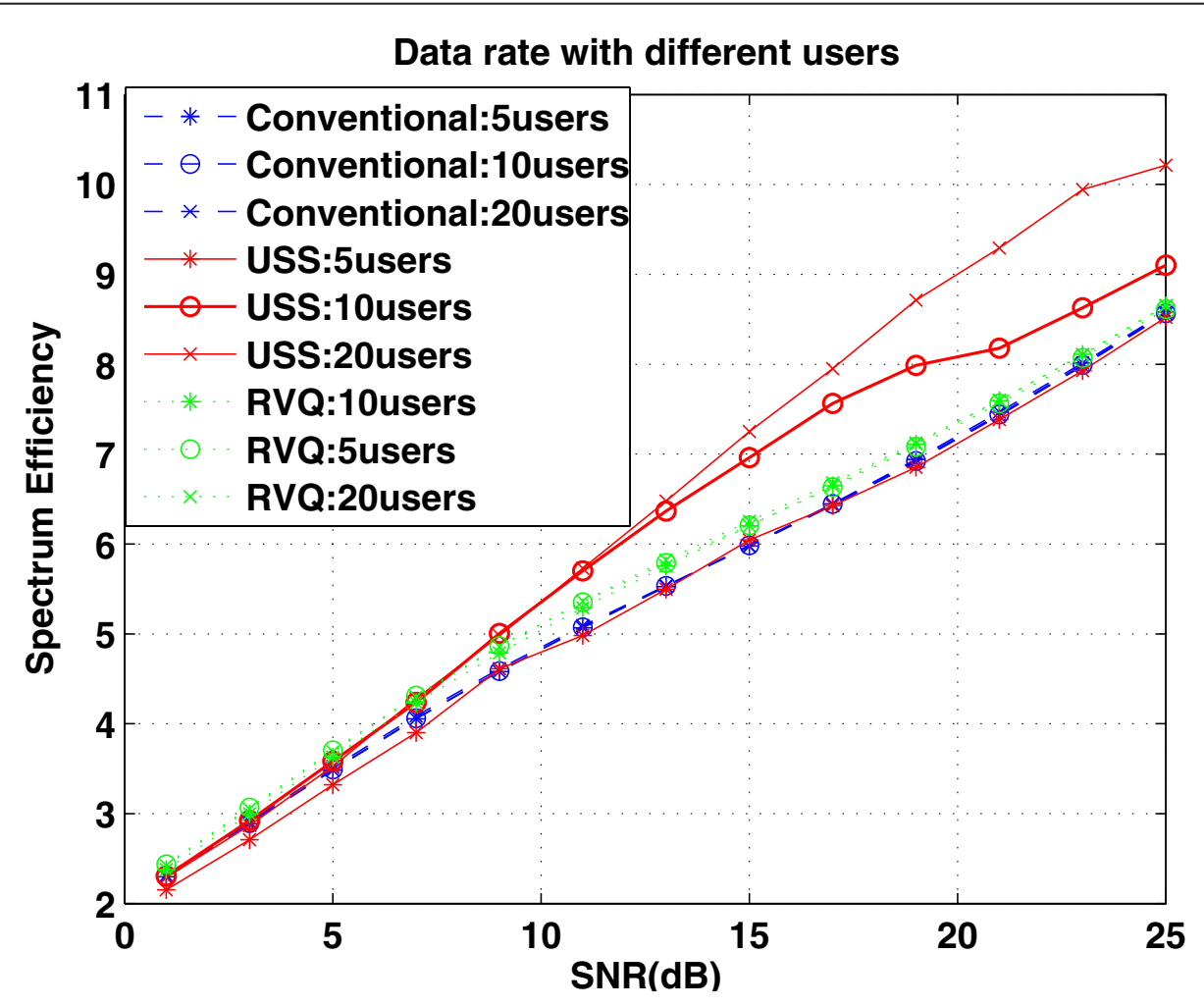

Figure 6 Throughput with different number of users. 


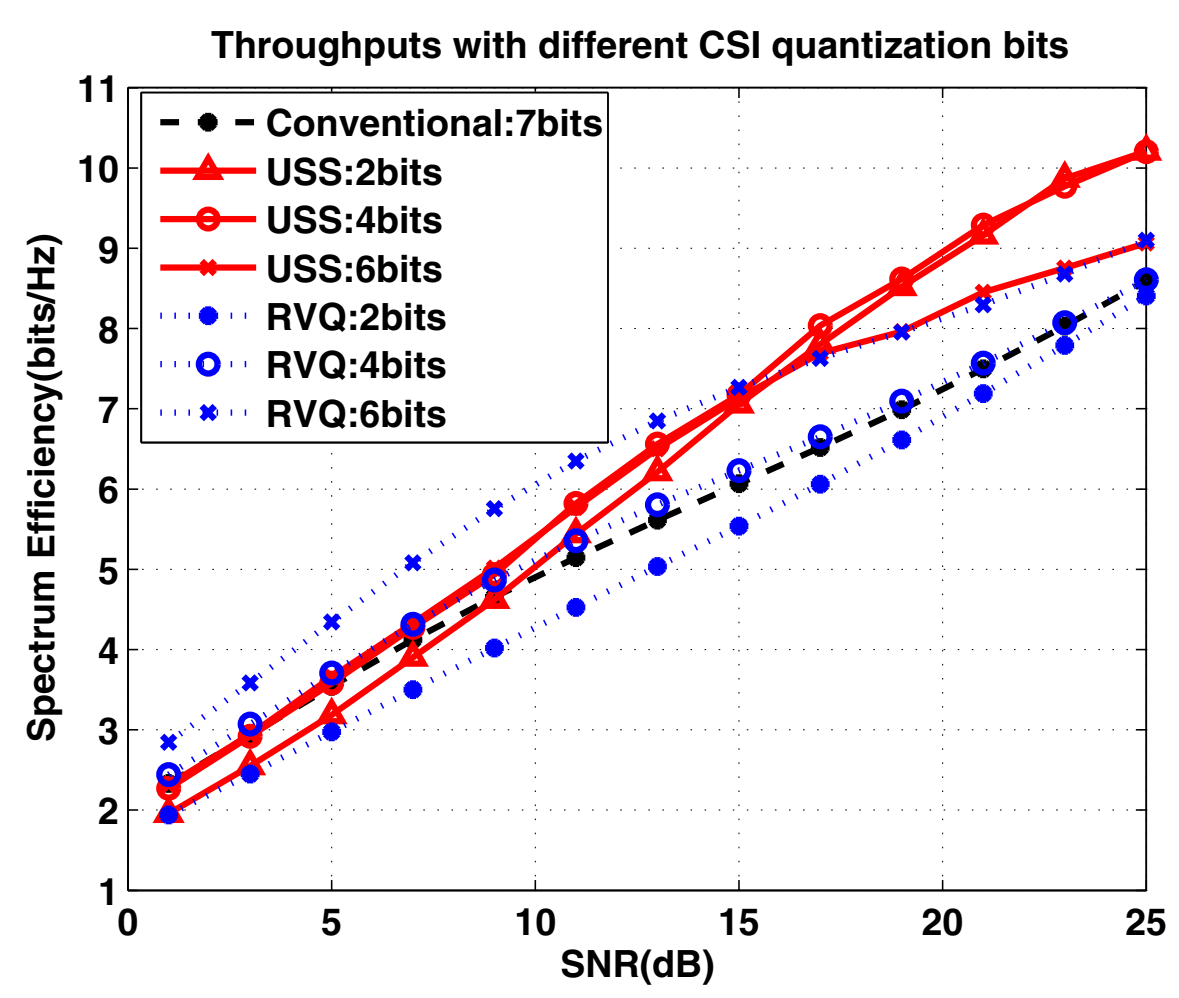

Figure 7 Throughput with different CSI quantization bits

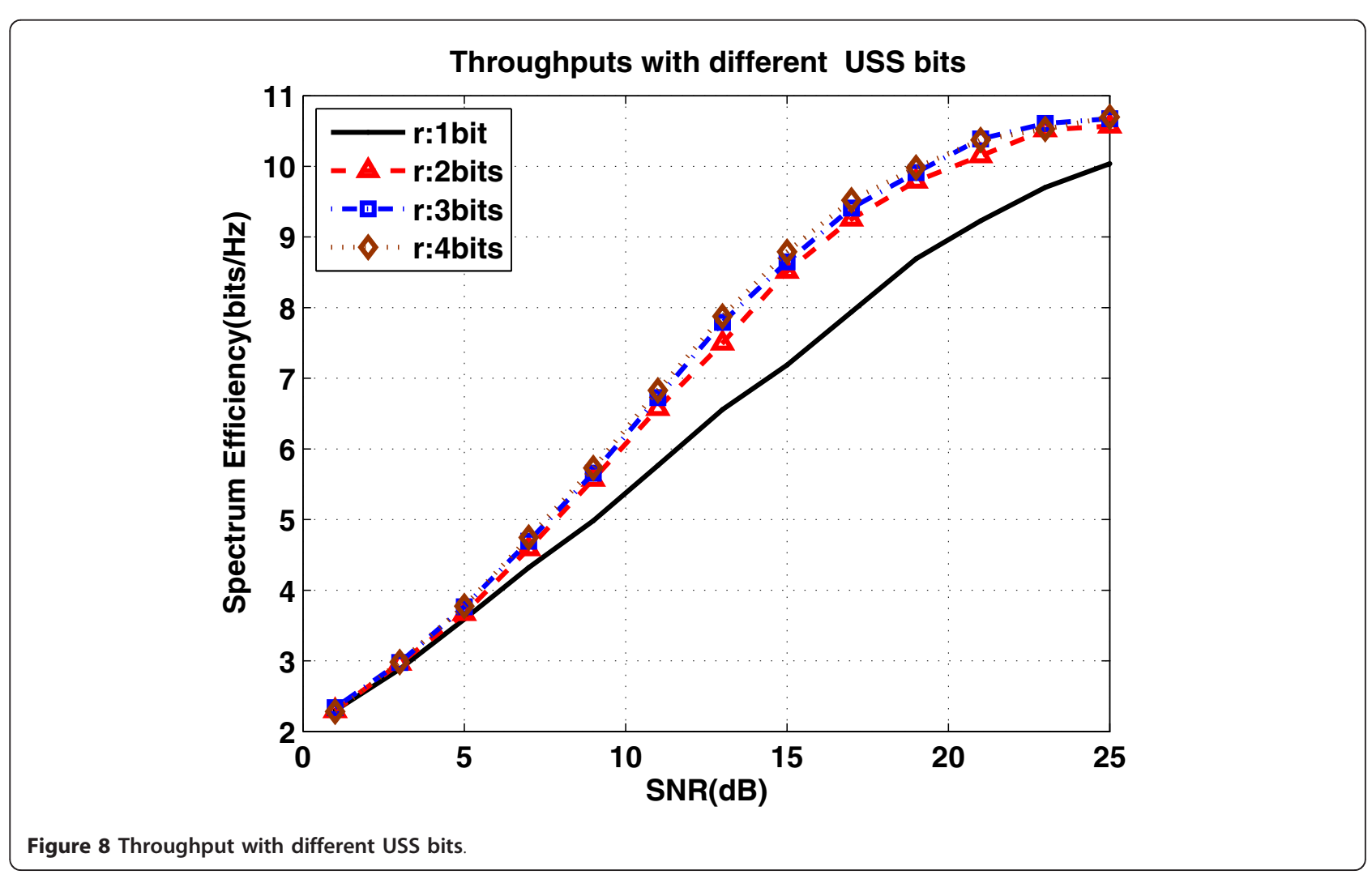


performance enhancement is larger while USS information increase from 1 bit to multiple bits. If the number of bits is more than 2, the enchantment is little. So, 2 bits (four orders) quantization of USS information is enough.

\section{Conclusion}

In this article, a novel USS feedback scheme and relative user selection procedure are proposed to avoid large inter-user interference in downlink ZF MU-MIMO for low rate fixed codebook feedback. The inter-user interference will largely decrease the MU performance gain in high SNR region and leads to the MU-MIMO throughput does not increase with the codebook size increasing. With the help of additional information, the proposed USS feedback scheme can avoid large interuser interference in ZF MU-MIMO transmission, and it can be used in various configurations such as different codebook type, different number of antennas, and different paired users. Simulation results show that the proposed USS feedback scheme is efficiency for users with very low CSI quantization bits and paired other users at high SNR region.

\section{Acknowledgements}

This study was supported by the National Natural Science Foundation of China Project (Grant No. 61001119, 61027003), the International Scientific and Technological Cooperation Program (Grant No. 2010DFA11060, S2010GR0902), and the National S\&T Major Program (No. 2009ZX03003-01102, No. 2009ZX03003-009).

\section{Competing interests}

The authors declare that they have no competing interests.

Received: 20 July 2011 Accepted: 10 January 2012

Published: 10 January 2012

\section{References}

1. E Telatar, Capacity of multi-antenna Gaussian channels. Eur Trans Telecommun. 10(6), 585-595 (1999). do:10.1002/ett.4460100604

2. P Viswanath, DNC Tse, Sum capacity of the vector Gaussian broadcast channel and uplink-downlink duality. IEEE Trans Inf Theory. 49(8), 1912-1921 (2003). doi:10.1109/TIT.2003.814483

3. M Costa, Writing on dirty paper. IEEE Trans Inf Theory. 29, 439-441 (1983). doi:10.1109/TIT.1983.1056659

4. H Weingarten, Y Steinberg, S Shamai, The capacity region of the Gaussian multiple-input multiple-output broadcast channel. IEEE Trans Inf Theory. 52(9), 3936-3964 (2006)

5. N Jindal, A Goldsmith, Dirty paper coding vs. TDMA for MIMO broadcast channels. IEEE Trans Inf Theory. 51(5), 1783-1794 (2005). doi:10.1109/ TIT.2005.846425

6. G Caire, S Shamai, On the achievable throughput of a multiantenna Gaussian broadcast channel. IEEE Trans Inf Theory. 49(7), 1691-1706 (2003). doi:10.1109/TIT.2003.813523

7. LU Choi, RD Murch, A transmit preprocessing technique for multiuser MIMO systems using a decomposition approach. IEEE Trans Wirel Commun. 3(1), 20-24 (2004). doi:10.1109/TWC.2003.821148

8. QH Spencer, AL Swindlehurst, M Haardt, Zero-forcing methods for downlink spatial multiplexing in multiuser MIMO channels. IEEE Trans Signal Proc. 52(2), 461-471 (2004). doi:10.1109/TSP.2003.821107

9. $\mathrm{N}$ Jindal, MIMO broadcast channels with finite rate feedback. IEEE Trans Inf Theory. 52(11), 5045-5059 (2006)
10. T Yoo, A Goldsmith, On the optimality of multiantenna broadcast scheduling using zero-forcing beamforming. IEEE J Sel Areas Commun. 24, 528-541 (2006)

11. J Lee, $N$ Jindal, High SNR analysis for MIMO broadcast channels: dirty paper coding vs. linear precoding. IEEE Trans Inf Theory. 53(12), 4487-4792 (2007)

12. J Sanchez-Garcia, L Soriano-Equigua, RW Heath, Quantized antenna combining for multiuser MIMO-OFDM with limited feedback. Signal Process Lett IEEE. 16(12), 1027-1030 (2009)

13. B Song, M Haardt, Effects of imperfect channel state information on achievable rates of precoded multi-user MIMO broadcast channels with limited feedback, in IEEE International Conference on Communications (ICC 2009), vol. 1. Dresden, Germany, pp. 1-5 (2009)

14. E Bjornson, D Hammarwall, B Ottersten, Exploiting quantized channel norm feedback through conditional statistics in arbitrarily correlated MIMO systems. IEEE Trans Signal Process. 57(10), 4027-4041 (2009)

15. S Zhou, Z Wang, GB Giannakis, Quantifying the power loss when transmit beamforming relies on finite-rate feedback. IEEE Trans Wirel Commun. 4(4), 1948-1957 (2005)

16. M Sharif, B Hassibi, On the capacity of MIMO broadcast channels with partial side information. IEEE Trans Inf Theory. 51(2), 506-522 (2005). doi:10.1109/TIT.2004.840897

17. H Shirani-Mehr, G Caire, Channel state feedback schemes for multiuser MIMO-OFDM downlink. IEEE Trans Commun. 57(9), 2713-2723 (2009)

18. T Yoo, N Jindal, A Goldsmith, Multi-antenna downlink channels with limited feedback and user selection. IEEE J Sel Areas Commun. 25(7), 1478-1491 (2007)

19. DJ Love, RW Heath, VKN Lau, D Gesbert, BD Rao, M Andrews, An overview of limited feedback in wireless communication systems. IEEE J Sel Areas Commun. 26(8), 1341-1365 (2008)

20. O Souihli, T Ohtsuki, Joint feedback and scheduling scheme for servicedifferentiated multiuser MIMO systems. IEEE Trans Wirel Commun. 9(2), 528-533 (2010)

21. F Liang, Y Maofan, G Ping, W Weiling, An efficient user scheduling scheme for MU-MIMO systems with limited feedback. in 2010 International Conference on Communications and Mobile Computing (CMC). 2, 348-35 (2010)

22. Z Chen, W Wang, M Peng, F Cao, Limited feedback scheme based on zeroforcing precoding for multiuser MIMO-OFDM downlink systems, in 2010 The 5th Annual ICST Wireless Internet Conference (WICON), vol. 1. (Chengdu, China, 2010), pp. 1-5

23. X Xia, G Wu, S Fang, S Li, SINR or SLNR: in successive user scheduling in mu-mimo broadcast channel with finite rate feedback. in 2010 International Conference on Communications and Mobile Computing (CMC). 2, 383-387 (2010)

24. M Trivellato, F Boccardi, F Tosato, User selection schemes for MIMO broadcast channels with limited feedback. in IEEE 65th Vehicular Technology Conference (VTC2007-Spring) (Dublin, Ireland). 1, 2089-2093 (2007)

25. A Bayesteh, AK Khandani, On the user selection for MIMO broadcast channels. IEEE Trans Inf Theory. 54(3), 1086-1107 (2008)

doi:10.1186/1687-1499-2012-7

Cite this article as: Li et al:: $R$ bits user selection switch feedback for zero forcing MU-MIMO based on low rate codebook. EURASIP Journal on Wireless Communications and Networking 2012 2012:7.

\section{Submit your manuscript to a SpringerOpen ${ }^{\circ}$ journal and benefit from:}

- Convenient online submission

- Rigorous peer review

- Immediate publication on acceptance

- Open access: articles freely available online

- High visibility within the field

- Retaining the copyright to your article

Submit your next manuscript at $>$ springeropen.com 